OPEN

SUBJECT AREAS:

APPLIED PHYSICS

TRANSFORMATION OPTICS

Received

18 September 2013

Accepted

5 December 2013

Published

20 December 2013

Correspondence and requests for materials should be addressed to

J.F.M. (jfmao@sjtu.

edu.cn) or Y.M.Z.

(ymzhu@usst.edu.cn)

*X.F. Zang contributed equally to this work.

\section{Overlapped optics induced perfect coherent effects}

\author{
Jian Jie Li' ', Xiao Fei Zang ${ }^{2 *}$, Jun Fa Mao', Min Tang ', Yi Ming Zhu² \& Song Lin Zhuang ${ }^{2}$
}

${ }^{1}$ Key Laboratory of Ministry of Education of China of Design and Electromagnetic Compatibility of High Speed Electronic System, Shanghai Jiao Tong University, Shanghai 200240, People's Republic of China, ${ }^{2}$ Engineering Research Center of Optical Instrument and System, Ministry of Education and Shanghai Key Lab of Modern Optical System, University of Shanghai for Science and Technology, No.516 JunGong Road, Shanghai 200093, People's Republic of China.

For traditional coherent effects, two separated identical point sources can be interfered with each other only when the optical path difference is integer number of wavelengths, leading to alternate dark and bright fringes for different optical path difference. For hundreds of years, such a perfect coherent condition seems insurmountable. However, in this paper, based on transformation optics, two separated in-phase identical point sources can induce perfect interference with each other without satisfying the traditional coherent condition. This shifting illusion media is realized by inductor-capacitor transmission line network. Theoretical analysis, numerical simulations and experimental results are performed to confirm such a kind of perfect coherent effect and it is found that the total radiation power of multiple elements system can be greatly enhanced. Our investigation may be applicable to National Ignition Facility (NIF), Inertial Confined Fusion (ICF) of China, LED lighting technology, terahertz communication, and so on.

$\mathrm{n}$ the normal case, two or more separated electromagnetic waves can be interfered with each other in free space, when all of them have the same frequency, vibration component and the constant initial phase $\mathrm{e}^{1,2}$. Taking two separated in-phase point sources for example, the corresponding total intensity at somewhere in the free space can be written as $I_{\text {total }}=\left(I_{1}+I_{2}\right)\left(1+\frac{2 \sqrt{I_{1} I_{2}}}{I_{1}+I_{2}} \cos \delta\right)$ with $\delta=k \cdot \Delta(k$ is the wave vector, and $\triangle$ is the optical path difference of these two point sources. $I_{1}$ and $I_{2}$ are the corresponding intensity of each point source, respectively.). For two separated identical in-phase point sources, the total intensity at free space can be expressed as $I_{\text {total }}=4 I_{0} \cos ^{2}\left(\frac{\delta}{2}\right)$ (Here, $\left.I_{1}=I_{2}=I_{0}\right)$. Obviously, only when $\Delta=m \lambda$, the total intensity of these two separated identical point sources is $4 I_{0}$. In this case $(\Delta=m \lambda)$, it can be considered that both of the point sources are overlapped with each other, resulting in the perfect coherent effects. One may ask is it possible to realize such a kind of perfect coherent without matching the condition of $\Delta=m \lambda$ and thus breaking the normal traditional perfect coherent condition? The answer is yes based on the transformation optics method. In the past few years, transformation optics is a new way to manipulate transmission path of electromagnetic waves. Many novel phenomena/devices such as invisible cloaks, field rotators, wave concentrators, beam splitter, and artificial wormholes/blackholes, and so on, have been designed and realized ${ }^{3-17}$.

Furthermore, by using transformation optics method, complementary media has been applied to design the anti-mirror effect where two separated objects with different shapes were overlapped with each other and appeared as only one, resulting in the overlapped optics effect ${ }^{18}$. Later, shifting media has been proposed in our previous work to realize the overlapped effect in which two separated objects with the same shape were overlapped with each other ${ }^{19}$. Recently, H. Y. Chen et al., have proposed a new conformal lens to realize the overlapped optics effect, and it can transform multiple in-phase sources into one ${ }^{20}$. Comparing with the above two kinds of overlapped optics devices, such a conformal lens can not only make multiple in-phase sources appear as only one but also make one active source appear as many in-phase sources. In a word, based on the complementary media, shifting media and conformal lens, two separated identical point sources can be effective overlapped together with each other in principle. Therefore, by these approaches, the amplitude of the two point in-phase sources can be increased by a factor of 2 , and the total intensity by a factor of 4 . However, all of these results are just shown in pure numerical simulations, lacking the corresponding experimental demonstration and theoretical explanation. Here, in this paper, we experimentally design the shifting illusion media by using the inductor-capacitor (L-C) transmission line network ${ }^{21}$. The corresponding shifting illusion media parameters are mimicked by controlling the parameters of the L-C unit cells, and the perfect coherent effects of two separated 


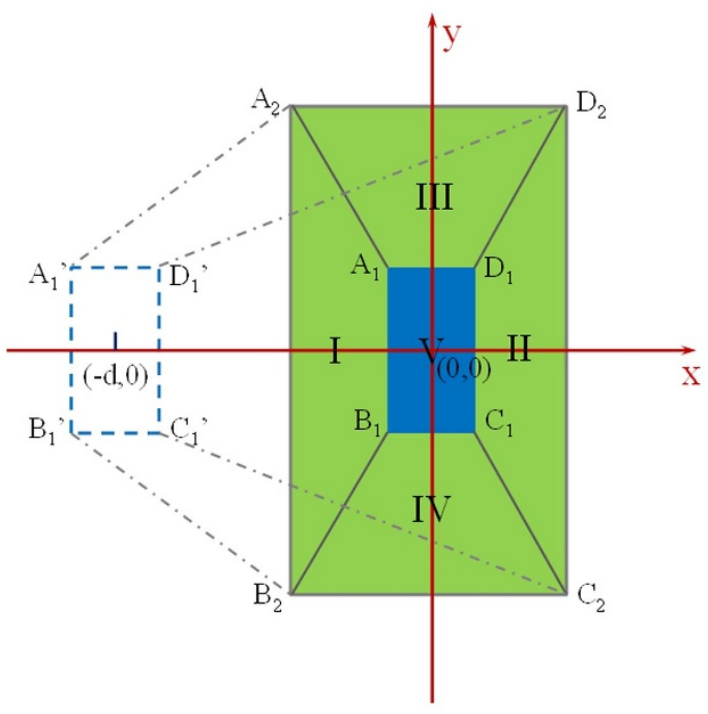

Figure 1 $\mid$ The principle of the shifting illusion media: Region V (blue region) covered with the shifting illusion media (green-yellow shell) is virtually shifted toward the left side with distance of $d$, and overlapped with the region encircled by the blue dashed border. Region I, II, III, and IV are the shifting media regions. The corresponding structure parameters are: $A_{1}\left(-a_{11}, b_{11}\right), B_{1}\left(-a_{11}, b_{11}\right), C_{1}\left(a_{11},-b_{11}\right), D_{1}\left(a_{11}, b_{11}\right), A_{2}\left(-a_{21}, b_{21}\right)$, $\mathrm{B}_{2}\left(-\mathrm{a}_{21},-\mathrm{b}_{21}\right), \mathrm{C}_{2}\left(\mathrm{a}_{21},-\mathrm{b}_{21}\right), \mathrm{D}_{2}\left(\mathrm{a}_{21}, \mathrm{~b}_{21}\right)$.

identical in-phase point sources is verified. Furthermore, a theoretical analysis is proposed to explain such a kind of perfect coherent effects. Our experimental results and theoretical analysis demonstrate that the fundamental perfect coherent condition is overturned for the first time based on the shifting media, and the radiation power of system can be greatly enhanced which may be applicable to NIF and ICF.

\section{Results}

Figure 1 shows the schematic of the shifting illusion media. A small blue region (Region $\mathrm{V}$ ) covered with a rectangular box of shifting illusion media (green-yellow region). In this situation, $\mathrm{A}_{1}{ }^{\prime} \mathrm{B}_{1}{ }^{\prime} \mathrm{B}_{2} \mathrm{~A}_{2}$ is folded into $A_{1} B_{1} B_{2} A_{2}$ due to the negative index material. Meanwhile, $\mathrm{A}_{1}{ }^{\prime} \mathrm{D}_{1}{ }^{\prime} \mathrm{D}_{2} \mathrm{~A}_{2}, \quad \mathrm{~B}_{1}{ }^{\prime} \mathrm{C}_{1}{ }^{\prime} \mathrm{C}_{2} \mathrm{~B}_{2}$, and $\mathrm{D}_{1}{ }^{\prime} \mathrm{C}_{1}{ }^{\prime} \mathrm{C}_{2} \mathrm{D}_{2}$ are mapped into $\mathrm{A}_{1} \mathrm{D}_{1} \mathrm{D}_{2} \mathrm{~A}_{2}, \mathrm{~B}_{1} \mathrm{C}_{1} \mathrm{C}_{2} \mathrm{~B}_{2}$, and $\mathrm{D}_{1} \mathrm{C}_{1} \mathrm{C}_{2} \mathrm{D}_{2}$, respectively. In other words, the central region (Region $\mathrm{V}$, free space with $\varepsilon=\mu=1$ ) is covered with the shifting illusion is shifted into the left side with a distance of $d$ and it is overlapped with that region encircled by the blue dash border. The Mathematical calculation of shifting media parameters will be discussed in details in Methods.

First, we give the numerical simulations of overlapped optics based on COMSOL Multiphysics ${ }^{\circledR}$, as shown in Fig. 2. Figure 2 (a) depicts the electric field distribution of only one point source with frequency of $3.68 \mathrm{GHz}$ in the free space. Figure 2 (b) shows the field distribution of two identical in-phase point sources in the free space. The electric field distribution in Fig. 2 (b) is inhomogeneous, which is quite different from the case of Fig. 2 (a). Due to the spatial coherence effect, these two separated identical in-phase point source appear strong/weak inhomogeneous field distribution. However, when the right point source coated with the shifting illusion media shown in Fig. 2(c) (the field distribution of in the dash box is shown in Fig. 2 (d)), the total field distribution of these two separated in-phase point sources outside the transformation region is homogeneous, and the corresponding electric field is enhanced with respect to the case of only one point source. In this case, the right point source covered with the shifting illusion media is shifted toward into left side and overlapped with the left source. Therefore, the total field distribution of these two separated in-phase point sources can be appeared both
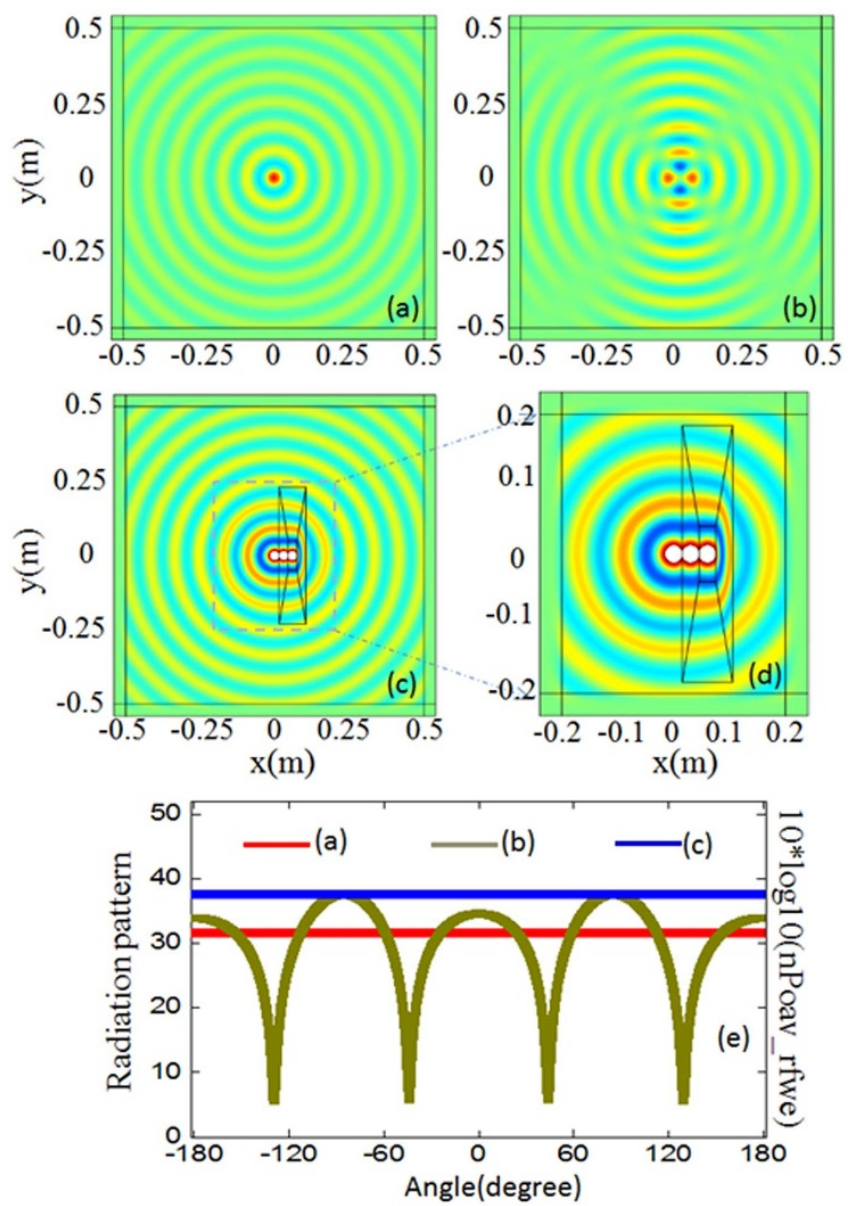

Figure 2 Field distribution of overlapped illusion optics: The excitation frequency of each in-phase source is $3.68 \mathrm{GHz}$. (a) Single point source located in $(0,0)$ in vacuum (free space) (b) Two identical point in-phase sources located at $(0,0)$ and $(0.06 \mathrm{~m}, 0)$, respectively. (c) Two identical point in-phase sources located at $(0,0)$ and $(0.06 \mathrm{~m}, 0)$ with right one covered with the shifting illusion media. (d) Field distribution in the dashed box of (c). (e) The corresponding power flow at $\mathrm{r}=0.4 \mathrm{~m}$ of (a) (red line), (b) (dark-yellow line), and (c) (blue line). Here, $\mathrm{a}_{11}=0.015 \mathrm{~m}$, $\mathrm{a}_{21}=0.045 \mathrm{~m}, \mathrm{~b}_{11}=0.05 \mathrm{~m}, \mathrm{~b}_{21}=0.23 \mathrm{~m}$, and $d=0.06 \mathrm{~m}$.

homogeneous and enhanced outside the transformation region. Figure 2 (e) shows the corresponding power flow of Fig. 2 (a), (b) and (c) at $r=0.4 \mathrm{~m}$. Obviously, the power flow of the two separated identical point sources in free space is inhomogeneous (dark-yellow line), while when the right point source covered with shifting illusion media, the power flow is homogeneous (blue line). Comparing with the blue line (two point source) and the red line (only one source), the power flow difference is about $6 \mathrm{~dB}$, which means that the total power of these two identical point sources is about quadruple (rather than double) to the case of just one point source, due to the perfect coherent effects.

Now, we experimentally demonstrate such a kind of perfect coherent effects, by using the $\mathrm{L}-\mathrm{C}$ transmission line network. A periodical $\mathrm{L}-\mathrm{C}$ transmission line network is designed and shown in Fig. 3 (a), to mimic the shifting illusion media. Unit cell of such L-C transmission line network are shown in Fig. 3 (b). According to the transmission line theory and electromagnetic theory, the L-C network equation can be mapped into the Maxwell's equation. For example, when $L_{x}=$ $L_{y}$, the L-C network can be considered as isotropic media with positive permeability and permittivity. Therefore, the shifting illusion media can be realized by controlling the parameter of inductor $\left(L_{x}\right.$, $\left.L_{y}\right)$ and capacitor $\left(C_{z}\right)$. In this paper, we just consider the long-wavelength limit, which means that the dimension of the unit cell is much 


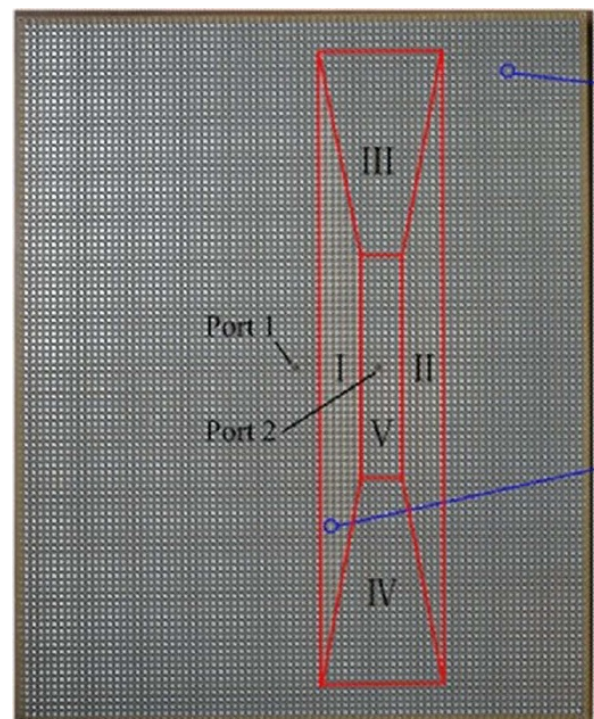

(a)
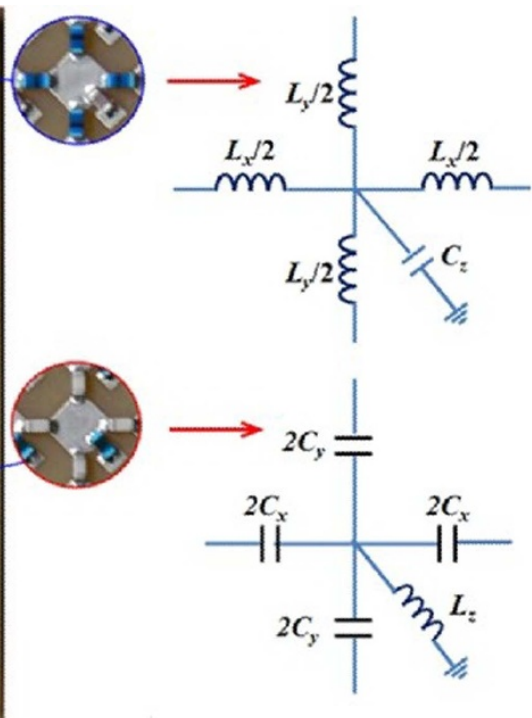

(b)

Figure $3 \mid$ (a) The testing sample with shifting illusion media in the box region. (b) Unit cell of L-C network: the up unit cell is corresponding to media with positive material parameters, and the down unit cell is corresponding to media with negative material parameters (the detail parameters are shown in Table I).

smaller than the wavelength of the excitation source, and the relation between the real material parameters and the L-C transmission line network can be written as follows:

$$
\begin{gathered}
\left\{\begin{array}{l}
\mu_{x}^{R}=L_{y} / \Delta \\
\mu_{y}^{R}=L_{x} / \Delta, \\
\varepsilon_{z}^{R}=C_{z} / \Delta
\end{array}\right. \\
\left\{\begin{array}{l}
\mu_{x}^{L}=-1 /\left(\omega^{2} C_{y} \Delta\right) \\
\mu_{y}^{L}=L_{R x} /\left(\omega^{2} C_{x} \Delta\right), \\
\varepsilon_{z}^{L}=-1 /\left(\left(\omega^{2} L_{z} \Delta\right)\right)
\end{array}\right.
\end{gathered}
$$

where $\Delta=5 \mathrm{~mm}$ is the unit cell length. "R" and "L" represent the positive permittivity/permeability and negative permittivity/permeability, respectively. The parameters in detail of our testing sample are listed in Table I. The fabrication and measurement of testing sample will be discussed in details in Methods. For the chosen configuration, the nondiagonal components of the shifting illusion media (which have comparatively small values in this study) are ignored in experiment to simplify the fabrication.

Fig. 4 (a) shows the simulated node voltage distribution of just one point source (with frequency of $65 \mathrm{MHz}$ ) excited at the node $(40,51$ ), based on the Agilent's advanced design system (ADS). Figure 4 (b) depicts the node voltage distribution of two separated identical inphase point sources excited at node $(40,51)$ and $(52,51)$, respectively. In this case, the right point source is coated by the shifting illusion media with the parameters in Table I. The node voltage distribution in Fig. 4 (b) is nearly the same as the corresponding field distribution in Fig. 2 (d), which means that the right point source covered with the

Table I | The unit cell parameters of the testing sample. Background represents the media outside the transformation region

\begin{tabular}{|ccccc} 
value region & $\begin{array}{c}\text { background vs } \\
\text { region III/IV/V }\end{array}$ & region II & value & $\begin{array}{c}\text { region } \\
\text { region I }\end{array}$ \\
\hline$L_{x}(\mathrm{nH})$ & 33 & 99 & $C_{x}(\mathrm{pF})$ & 222 \\
$L_{y}(\mathrm{nH})$ & 33 & 11 & $C_{y}(\mathrm{pF})$ & 222 \\
$C_{z}(\mathrm{pF})$ & 27 & 82 & $L_{z}(\mathrm{nH})$ & 200 \\
\hline
\end{tabular}

shifting illusion media is overlapped with the left one. Comparing Fig. 4 (a) with Fig. 4 (b), it can be found that the node voltage distribution of the two identical point sources is significant enhanced, although both of them have the similar distribution profile. The experimental result of voltage distribution is shown in Fig. 4 (c). The measured voltage distribution in Fig. 4 (c) is well consistent with the simulation results in Fig. 4 (b) and Fig. 2 (d).

\section{Discussion}

Let's look at the power of the test sample leaking into the right domain. Both the simulated and measured results are given in Fig. 4(d). Similar to the conclusion of Fig. 2(e), the power of the two overlapped identical point sources is quadruple of the case of just one point source due to the perfect coherent effect. We explain this phenomenon from antenna point of view as follows. According to electromagnetic theory, the radiated power of single antenna is calculated as:

$$
P_{s}=I^{2} R_{r}
$$

where I and $R_{r}$ are the root-mean-square value of excitation current and radiation resistance of antenna, respectively. The total radiated power of an antenna array with $\mathrm{N}$ such element is:

$P_{\text {array }}=\left(\begin{array}{lllll}I_{1} & I_{2} & I_{3} & \ldots & I_{N}\end{array}\right)\left(\begin{array}{ccccc}R_{11} & R_{12} & R_{13} & \ldots & R_{1 N} \\ R_{21} & R_{22} & R_{23} & \ldots & R_{2 N} \\ R_{31} & R_{32} & R_{33} & \ldots & R_{3 N} \\ \vdots & \vdots & \vdots & \ddots & \vdots \\ R_{N 1} & R_{N 2} & R_{N 3} & \ldots & R_{N N}\end{array}\right)\left(\begin{array}{c}I_{1}^{*} \\ I_{2}^{*} \\ I_{3}^{*} \\ \vdots \\ I_{N}^{*}\end{array}\right)(4)$

where $I_{i}(i=1,2 \ldots, N)$ is the root-mean-square value of excitation current of $i^{\text {th }}$ element and $I_{i}^{*}(i=1,2 \ldots, N)$ is the complex conjugate of $I_{i}(i=1,2 \ldots, N), R_{i i}(i=1,2 \ldots, N)$ is the radiation self-resistance of $i^{\text {th }}$ element, $R_{i j}(i, j=1,2 \ldots, N, i \neq j)$ is the radiation mutualresistance of $i^{\text {th }}$ element to $j^{\text {th }}$ element. From the above analysis, the performance of the in-phase elements placed in different positions is same as each other when the proposed shifting illusion media is placed around all the in-phase elements, therefore $R_{i i}=R_{i j}=R_{r}(i$, $j=1,2 \ldots, N)$. If $I_{1}=I_{2}=\cdots=I_{N}=I$, the total radiation power of the array becomes: 

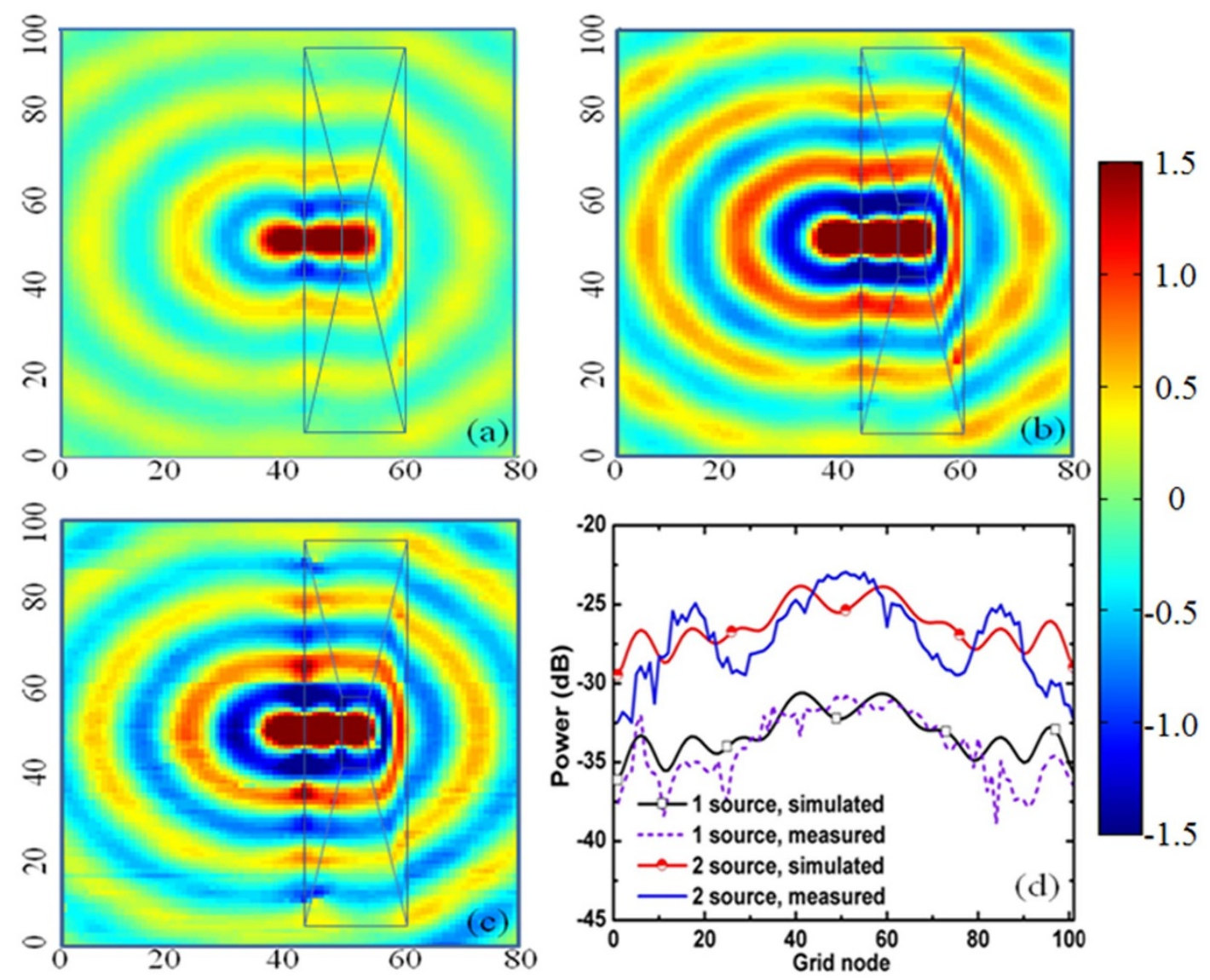

Figure $4 \mid$ Simulated and measured node voltage distribution of the shifting illusion media. The frequency of the excitation source is $65 \mathrm{MHz}$. (a) One excitation source (simulation) located at the node of $(40,51)$. (b) Two identical in-phase excitation sources located at the node of (40, 51), and (52, 51), respectively (simulation). (c) Two identical in-phase excitation sources located at the node of $(40,51)$, and (52, 51), respectively (experiment). (d) The corresponding leaked power into the right boundary of the sample.

$$
P_{\text {array }}=N^{2} \cdot I^{2} R_{r}=N^{2} \cdot P_{s}
$$

Thus, the total radiation power of an antenna array with two inphase elements is 4 times $(6 \mathrm{~dB})$ that of single antenna. This conclusion consists with that of Fig. 2(e) and Fig. 4(d). If this technology is applied to NIF, the total radiation power of 192 beam lines of NIF would be 36864 times that of single laser beam lines and it may be closer to the condition of achieving fusion ignition.

In conclusion, we have realized a shifting illusion media to investigate the perfect coherent effects. The traditional coherent condition seemed insurmountable was totally breakthrough by using the shifting media for the first time. Two identical microwave in-phase sources with one of them covered with the shifting illusion media are found to be overlapped with each other, leading to the perfect coherent effects without needing the conventional condition of $\Delta=$ $m \lambda$. The theoretical analysis, numerical simulations and experimental measurements are given to demonstrate such a kind of perfect coherent effects, and it is found the radiation power of multiple elements system can be greatly enhanced. This technology is possible to eliminate the interference and improve the brightness of LED, and may be applicable to the situation of needing high radiation power, such as NIF, ICF of China, antenna array, and so on.

\section{Methods}

Mathematical calculation of shifting media. The corresponding coordinates in Fig. 1 are: $A_{1}\left(-a_{11}, b_{11}\right), B_{1}\left(-a_{11}, b_{11}\right), C_{1}\left(a_{11},-b_{11}\right), D_{1}\left(a_{11}, b_{11}\right), A_{2}\left(-a_{21}, b_{21}\right)$, $\mathrm{B}_{2}\left(-\mathrm{a}_{21},-\mathrm{b}_{21}\right), \mathrm{C}_{2}\left(\mathrm{a}_{21},-\mathrm{b}_{21}\right), \mathrm{D}_{2}\left(\mathrm{a}_{21}, \mathrm{~b}_{21}\right)$. According to the transformation optics, the coordinate transformation between the physical space and the virtual space can be given below:

$$
\begin{aligned}
& \text { Region I : } \quad x^{\prime}=\frac{\left(a_{21}-a_{11}\right) x+d a_{21}}{a_{21}-a_{11}-d}, \quad y^{\prime}=y, \quad z^{\prime}=z \\
& \text { Region II : } \quad x^{\prime}=\frac{\left(a_{21}-a_{11}\right) x+d a_{21}}{a_{21}-a_{11}+d}, \quad y^{\prime}=y, \quad z^{\prime}=z . \\
& \text { Region III : } \quad x^{\prime}=x+\frac{d\left(b_{21}-y\right)}{b_{21}-b_{11}}, \quad y^{\prime}=y, \quad z^{\prime}=z \\
& \text { Region IV : } \quad x^{\prime}=x+\frac{d\left(b_{21}+y\right)}{b_{21}-b_{11}}, \quad y^{\prime}=y, \quad z^{\prime}=z
\end{aligned}
$$

The relative permittivity $\overline{\bar{\varepsilon}}$ and permeability $\overline{\bar{\mu}}$ of the shifting illusion media are: In Region I:

$$
\overline{\bar{\varepsilon}}=\overline{\bar{\mu}}=\left[\begin{array}{ccc}
\frac{a_{21}-a_{11}}{a_{21}-a_{11}-d} & 0 & 0 \\
0 & \frac{a_{21}-a_{11}-d}{a_{21}-a_{11}} & 0 \\
0 & 0 & \frac{a_{21}-a_{11}-d}{a_{21}-a_{11}}
\end{array}\right]
$$

In Region II:

$$
\overline{\bar{\varepsilon}}=\overline{\bar{\mu}}=\left[\begin{array}{ccc}
\frac{a_{21}-a_{11}}{a_{21}-a_{11}+d} & 0 & 0 \\
0 & \frac{a_{21}-a_{11}+d}{a_{21}-a_{11}} & 0 \\
0 & 0 & \frac{a_{21}-a_{11}+d}{a_{21}-a_{11}}
\end{array}\right]
$$




$$
\overline{\bar{\varepsilon}}=\overline{\bar{\mu}}=\left[\begin{array}{ccc}
1+\left(\frac{d}{b_{21}-b_{11}}\right)^{2} & -\frac{d}{b_{21}-b_{11}} & 0 \\
-\frac{d}{b_{21}-b_{11}} & 1 & 0 \\
0 & 0 & 1
\end{array}\right]
$$

In Region IV:

$$
\overline{\bar{\varepsilon}}=\overline{\bar{\mu}}=\left[\begin{array}{ccc}
1+\left(\frac{d}{b_{21}-b_{11}}\right)^{2} & \frac{d}{b_{21}-b_{11}} & 0 \\
\frac{d}{b_{21}-b_{11}} & 1 & 0 \\
0 & 0 & 1
\end{array}\right]
$$

Fabrication of testing sample. The testing sample is fabricated on a grounded flame retardant 4 (FR4) substrate with $1 \mathrm{~mm}$-thickness and dielectric constant of $\varepsilon_{r}=4.3$. The whole sample consists of 81 grid nodes along $\mathrm{x}$ direction and 101 grid nodes along y direction. The interval between two nodes is $5 \mathrm{~mm}$. The surface-mounted capacitors and inductors of the testing sample are 0603 package size. The nodes in the region I consist of four surface-mounted capacitors in series and one inductor in shunt to the ground by a via-hole. The nodes of other regions consist of four surfacemounted inductors in series and one capacitor in shunt to the ground by a via-hole. The outer boundary of the structure is truncated with Bloch impedances.

Measurement of testing sample. Agilent E5071C VNA is applied to measure transmission coefficient of each grid node, which is proportional to the voltage and electric field of node. Port 1 of VNA is connected to the input port of a 3-port divider, and the two output ports of the divider are connected to node $(40,51)$ and $(52,51)$ respectively, providing two identical excitation sources. Port 2 of VNA connected to a high-impedance amplifier scans over the surface of our sample to obtain the voltage of each node.

1. Born, M. \& Wolf, E. [Interference of two monochromatic waves] Principles of Optics: Electromagnetic theory of propagation, interference and Diffraction of Light 257-259 (A. Wheaton \& Co. Ltd, Great Britain, 1980).

2. Jackson, J. D. [Superposition of waves in one dimension, group velocity] Classical Electrodynamics 299-302 (John Wiley \& Sons, New York, 1998).

3. Pendry, J. B., Schurig, D. \& Smith, D. R. Controlling electromagnetic fields. Science 312, 1780-1782 (2006).

4. Leonhardt, U. Optical conformal mapping. Science 312, 1777-1780 (2006).

5. Schurig, D. et al. Metamaterial electromagnetic cloak at microwave frequencies. Science 314, 977-980 (2006).

6. Liu, R. et al. Broadbsnd ground-plane cloak. Science 323, 366-369 (2009).

7. Valentine, J., Li, J., Zentgraf, T., Bartal, G. \& Zhang, X. An optical cloak made of dielectrics. Nature Materials 8, 568-571 (2009).

8. Chen, H. Y., Chan, C. T. \& Shen, P. Transformation optics and metamaterials. Nature Materials 9, 387-396 (2010).

9. Zhou, F. et al. Hiding a Realistic Object Using a Broadband Terahertz Invisibility Cloak. Sci. Rep. 1, 78; DOI:10.1038/srep00078 (2011).
10. Liang, D. et al. Robust large dimension terahertz cloaking. Advanced Materials 24, 916-921 (2012).

11. Ma, H. F. \& Cui, T. J. Three-dimensional broadband ground-plane cloak made of metamaterials. Nature Communications 1, 21 (2010).

12. Liu, Y. M., Zentgraf, T., Bartal, G. \& Zhang, X. Transformational Plasmon optics. Nano Letters 10, 1991-1997 (2000).

13. Rahm, M., Schurig, D., Roberts, D. A., Cummer, S. A. \& Smith, D. R. Design of electromagnetic cloaks and concentrators using form-invariant coordinate transformations of Maxwell's equations. Photonics and NanostructuresFundamentals and Applications 6, 87-95 (2008).

14. Rahm, M., Cummer, S. A., Schurig, D., Pendry, J. B. \& Smith, D. R. Optical design of reflectionless complex media by finite embedded coordinate transformation. Physical Review Letters 100, 063903 (2008).

15. Greenleaf, A., Kurylev, Y., Lassas, M. \& Uhlmann, G. Electromagnetic wormholes and virtual magnetic monopoles from metamaterials. Physical Review Letters $\mathbf{9 9}$, 183901 (2007).

16. Zhang, B., Luo, Y., Liu, X. \& Barbastathis, G. Macroscopic invisibility cloak for visible light. Physical Review Letters 106, 033901 (2011).

17. Chen, H. Y., Li, M. \& Miao, R. X. Transformation optics that mimics the system outside a Schwarzschild black hole. Opt. Express. 18, 15183-15188 (2010).

18. Xu, Y. D., Du, S. W., Gao, L. \& Chen, H. Y. Overlapped illusion optics: a perfect lens brings a brighter feature. New. J. Phys. 13, 023010 (2011).

19. Zang, X. F. \& Jiang, C. Overlapped optics, illusion optics, and an external cloak based on shifting media. J. Opt. Soc. Am. B. 28, 1994 (2011).

20. Chen, H. Y., Xu, Y. D., Li, H. \& Tyc, T. Playing the tricks of numbers of light sources. New. J. Phys. 15, 093034 (2013).

21. Li, C. et al. Experimental realization of a circuit-based broadband illusion-optics analogue. Physical Review Letters 105, 233906 (2010).

\section{Acknowledgments}

This work was supported by the National Natural Science Foundation of China under Grant Nos. 61327803, 61138001. We thank for Professor Chao Li for helpful suggestions and discussions.

\section{Author contributions}

J.J.L. and M.T. performed ADS simulations and fabricated the device. J.J.L. wrote the part of discussion and method. X.F.Z. proposed the shifting media theory, performed COMSOL Multiphysics ${ }^{\circledR}$ simulations and wrote the most part of the paper. J.F.M. conceived the experiments and supervised the measurement. Y.M.Z. and S.L.Z. supervised the theoretical analysis, measurement and writing.

\section{Additional information}

Competing financial interests: The authors declare no competing financial interests.

How to cite this article: Li, J.J. et al. Overlapped optics induced perfect coherent effects. Sci. Rep. 3, 3569; DOI:10.1038/srep03569 (2013)

(c) (i) (2) This work is licensed under a Creative Commons AttributionBY NC SA NonCommercial-ShareAlike 3.0 Unported license. To view a copy of this license, visit http://creativecommons.org/licenses/by-nc-sa/3.0 